Voix et Images

volxetimages

\title{
L'écriture comme exploration : propos, envolées et digressions. Entretien avec Normand Chaurette
}

\section{Pascal Riendeau}

Volume 25, numéro 3 (75), printemps 2000

Normand Chaurette

URI : https://id.erudit.org/iderudit/201495ar

DOI : https://doi.org/10.7202/201495ar

Aller au sommaire du numéro

Éditeur(s)

Université du Québec à Montréal

ISSN

0318-9201 (imprimé)

1705-933X (numérique)

Découvrir la revue

Citer ce document

Riendeau, P. (2000). L'écriture comme exploration : propos, envolées et digressions. Entretien avec Normand Chaurette. Voix et Images, 25(3), 436-448. https://doi.org/10.7202/201495ar d'utilisation que vous pouvez consulter en ligne.

https://apropos.erudit.org/fr/usagers/politique-dutilisation/ 


\title{
L'écriture comme exploration: propos, envolées et digressions. Entretien avec Normand Chaurette
}

\author{
Pascal Riendeau, Université de Montréal
}

V et I: On ne fera pas, si vous le voulez bien, de bilan de votre carrière ni même de parcours systématique de votre œuvre depuis Rêve d'une nuit d'hôpital, jouée pour la première fois sur la scène en 1980 et publiée la même année. Cependant, après vingt ans, vous avez réussi à vous tailler une place parmi les plus enviables chez les dramaturges québécois contemporains. Comment évaluez-vous cette place aujourd'hui?

N. C. : On commence seulement à parler des années quatre-vingt. Évidemment, 1980, c'est un peu comme ma date de naissance. Vingt ans, ce n'est pas beaucoup. J'en viens quand même à réfléchir à l'idée d'un parcours. C'est comme si un pari avait été posé au départ. Je n'en faisais pas une question de vie ou de mort, mais c'était quand même un pari assez sérieux: me trouver un espace d'écrivain dans le théâtre québécois dont on enseignait l'oralité,-le message non équivoque, la faillite poétique et l'audace qui tendait à vouloir le rendre populaire par l'improvisation, l'irrévérence sociale, voire un dédain de la littérature. Je me souviens d'un cours de théâtre ayant pour titre "Démystification du joual ". L'avenue des créations collectives avait conduit à de grandes autoroutes, mais on en voyait désormais l'impasse. J'ai commencé à écrire à l'université. En fait, la dernière chose qui m'intéressait, à ce moment-là, c'était le théâtre. Ce dont on rêve à 22 ans, quand on écrit en marge des lectures d'Hubert Aquin, c'est de devenir un écrivain québécois. Je n'envisageais pas une carrière de dramaturge. D'ailleurs, le mot "dramaturgie" ne s'employait pas en dehors du milieu théâtral, que je n'ai rencontré que plus tard. Le théâtre, c'est... la vie qui m'a rattrapé. C'est vraiment le hasard qui m'a fait gagner un prix à Radio-Canada sur la présentation d'une nouvelle, qui a été transformée en scénario et qui m'a conduit au prix Gilson pour Rêve d'une nuit d'bôpital en 1976. Quatre ans plus tard, cette pièce est adaptée pour la scène et représentée au Théâtre de Quat'Sous. Cette création participe d'un fait nouveau qu'on observe dans le paysage théâtral au début de 1980. Nous sommes trois ou quatre à être montés au cours de la même saison, de qui on dit alors: il y a une rupture fondamentale entre 
une oralité et une écriture. Panique à Longueuil de René-Daniel Dubois, c'est un acte d'écriture. Les pièces de Michel Marc Bouchard sont des textes. Il y a donc apparition d'une génération spontanée en 1980, encouragée a priori par les intellectuels. C'est vrai qu'en 1985, je suis obligé de constater que je suis un peu le seul qui continue d'écrire avec une syntaxe, une attention à la musique de la langue et que tranquillement les auteurs optent pour la voie du contenu et d'une théâtralité efficace, laquelle semble d'emblée conduire au succès de Being at home with Claude de Dubois et des Feluettes de Bouchard deux ans plus tard. Dans mon cas, 1987 et 1988 auront été des années de réflexion au regard du peu de popularité de mes pièces telles qu'on les représente. Entre 1986, l'année de Fragments d'une lettre d'adieu lus par des géologues, et 1991, l'année de la publication des Reines, il ne se produit rien sur le plan de ma carrière. Il se passe beaucoup de textes, beaucoup d'approfondissements, mais pas de choses importantes sur scène.

V et I: Pourtant, il y a eu Scènes d'enfants en $1988 \ldots$

N. C. : Voilà : il y a le roman, qui est une façon d'interroger le théâtre. Les gens se rendent compte que ce n'est pas tellement rentable de monter Chaurette; il y a des impératifs économiques dans les théâtres, et je suis le premier à les encourager en leur disant: "Vous avez raison, je suis un cadeau empoisonné, je ne fais que des flops..." Après, avec Les reines, le vent a tourné. Shakespeare occupe une place énorme dans le théâtre québécois vers la fin des années quatre-vingt. On se demande souvent si l'on est pas un peu opportuniste, mais la vibration shakespearienne s'est aussi manifestée dans ma démarche. On m'avait demandé de traduire Richard III; j'étais à l'aise avec les personnages féminins, alors j'ai fait Les reines. En résumé, on peut affirmer qu'il y a deux décennies distinctes dans ma création. À partir de 1991, c'est une autre histoire complètement. Avant, c'était tout nouveau, du Chaurette. Après, ça devient un auteur responsable d'une façon de faire, quelqu'un aux prises avec une construction et qui s'est développé un lectorat, plus qu'un public.

V et I : Vous avez parlé de René-Daniel Dubois et de Michel Marc Bouchard. On a très (trop?) souvent associé votre écriture dramatique aux leurs en disant qu'il s'agissait de la nouvelle dramaturgie des années quatre-vingt ou d'une dramaturgie postmoderne. Vous sentez-vous proche de ces deux dramaturges qui ont à peu près le même âge que vous?

N. C.: Non, et je crois bien que là-dessus, nous sommes tous les trois d'accord. On nous a beaucoup associés, de façon je dirais inévitable, à cause de cette coïncidence. On a cessé de le faire après 1988, alors que les Cabiers de théâtre Jeu ont publié un numéro sur le théâtre et l'homosexualité, dont nous avions dénoncé le peu de rigueur. Cette façon-là de nous unir par une orientation sexuelle, qui depuis longtemps avait cessé d'être un tabou, manquait de pertinence. Était-ce parce que nous avions 
chacun écrit notre pièce gay? Si c'est le cas, c'est dommage. Provincetown Playhouse, juillet 1919, j'avais 19 ans n'est pas une piège gay; Being at bome with Claude n'est pas une piège gay, mais un texte mettant en scène l'homosexuel qui, sur le plan humain, explore une blessure en la rendant universelle. Dans Les feluettes, c'est une communauté homosexuelle qui se retrouve en tant qu'élément historique, ni plus ni moins que dans Provincetown Playbouse. Chez moi, l'explication homosexuelle ne fait que servir une intrigue policière à la fin. Charles Charles parle de son amant au sens décadent du terme. C'est l'homosexualité de 1919, ce n'est pas un texte de réhabilitation de l'homosexualité.

$\mathbf{V}$ et I : Bien au-delà de la thématique homosexuelle, je fais un rapprochement entre votre écriture et celles des deux autres, surtout celle de Dubois: un éclatement des formes dramatiques, une confusion spatiotemporelle, une interrogation sur le sujet...

N. C. : Les oranges sont vertes étaient écrites depuis longtemps. Gauvreau était passé avant nous. On avait l'habitude de vouloir résumer le théâtre québécois à ses deux grandes tendances: Marcel Dubé dans les années soixante et Michel Tremblay dans les années soixante-dix. On négligeait les travaux plus souterrains de Gurik, Sauvageau, Loranger, quand ils ne s'inscrivaient pas dans un camp ou dans l'autre. De plus, on parle de tout ça comme si ça avait été prémédité! Nous ne sommes pas arrivés ni l'un ni l'autre avec l'idée de faire un théâtre éclaté. J'irais plus loin: mon manque d'expérience, mon peu d'habileté à construire une histoire qui se tienne favorisait cet éclatement. Le thêâtre m'offrait la possibilité d'être approximatif sur la question de l'efficacité. Un texte comme Rêve d'une nuit d'bôpital n'aurait pas été acceptable pour une télésérie.

V et I : Jusqu'au milieu des années quatre-vingt-dix, on demandait: vous savez, Chaurette, l'auteur de Provincetown Playhouse? Ah oui!, répondaiton. Maintenant, Provincetown Playbouse semble de plus en plus reléguée à une autre époque, je vous ai même entendu dire qu'il s'agissait d'une pièce "d'adolescent" (dans le programme de la saison 1999-2000 du Théâtre du Nouveau Monde, on vous présente comme l'auteur des Reines et du Passage de l'Indiana). Selon moi, Provincetown Playbouse demeure un des textes les plus fascinants que vous ayez écrits. Cela dit, est-ce que Provincetown Playbouse a été, comme Le libraire de Bessette ("Maudit Libraire! „, disait-il), un obstacle pour vos textes ultérieurs, toujours jugés à l'aune de Provincetown Playbouse?

N. C. : Non. Pas du tout. J'ai toujours été fier de cette pièce. Même s'il est vrai qu'on ne refait pas deux fois Provincetown Playbouse. Mais ce texte ayant été plus commenté que les autres, j'ai craint qu'il ne porte ombrage à des travaux moins efficaces au strict plan dramaturgique. Provincetown Playbouse avait l'avantage - qui s'est transformé pour moi en désavantage - d'être une pièce sur le théâtre. Je ne voulais pas réécrire une 
pièce qui parle du théâtre parce que je me méfiais de la lourdeur éventuelle du propos, et je ne suis pas porté vers une ambition théorique. Provincetown Playhouse demeure un texte écrit en dix jours, en 1978. Puis, le texte est allé dans les tiroirs et il y est resté jusqu'en 1982, parce que la seule personne qui l'avait lu m'avait dit que ce n'était pas très fort et que ça prenait trop de culture américaine pour lire la pièce. Je ne voulais pourtant pas écrire une pièce savante. Je me souviens d'avoir rêvé de l'Acteur avec un grand A, de l'acteur mûr, pour jouer Charles Charles. À vingt-quatre ans, trente-huit ans, c'est vieux! C'est pour ça probablement que j'en ai parlé comme d'une pièce d'adolescent!... Cette histoire brutale, violente, sur le meurtre d'un enfant pour servir une vengeance d'amoureux, reste pour moi ma pièce en joual. Je m'explique. Dans Provincetown Playbouse, on ne retrouve pas de morceau d'écriture à proprement parler (j'exclus les variantes poétiques que l'éditeur avait tenu à publier à la fin du livre); je poursuis une histoire, je cherche un coupable, une conclusion. Dans mes autres pièces, tout fonctionne sur un modèle plus musical, les personnages ont à dire des monologues où je me permets des envolées que je suis le premier à traiter de coquetteries. J'essaie de lutter contre ça, je crois qu'il s'agit d'une faiblesse, pour ne pas parler de complaisance. Mais dans Provincetown Playbouse, pièce plus réaliste sur le plan du langage, les personnages sont jeunes, brutaux, ils n'ont pas la parole facile. J'avais été très marqué par le personnage de Claire Lannes dans L'amante anglaise de Marguerite Duras. Elle est traquée, folle, elle n'a pas les mots. Toute la beauté du drame vient de ce qu'elle ne trouve pas les mots pour répondre à l'interrogatoire. J'envie beaucoup cette dureté du manque à dire chez les auteurs contemporains, je pense à Koltès.

V et I : Les références à Duras et à Koltès m'incitent à aborder avec vous le rapport entre le théâtral et le romanesque. On remarque dans vos textes dramatiques une tentation du narratif. Après Scènes d'enfants, êtesvous toujours attiré par la forme romanesque? Est-ce que le mini-recueil de nouvelles intitulé Le pont du Gard vu de nuit nous laisse présager autre chose du côté de la nouvelle ou du récit en général?

N. C. : Il existe effectivement un immense chantier d'écriture qui s'appelle Ponts et légendes, qui verra probablement le jour. L'attente n'est pas créée là-dessus. J'en parle très rarement; c'est un lieu de dialogue entre mon éditeur (Pierre Filion) et moi. C'est un lieu privilégié où je ne sens aucune autre contrainte que celle que je m'impose. Ce sont des ponts et légendes, et c'est autobiographique; je ne me censure aucunement et j'essaie d'atteindre une sorte de vérité qui est différente de celle qu'on met dans la bouche d'un personnage de théâtre. C'est un roman qui est écrit au "je " et qui alterne entre le propos banal, le bavardage quotidien, et aussi la réflexion plus approfondie sur l'écriture. La métaphore centrale est celle du pont, qui unit les rives, les êtres et les choses. Je suis un 
voyageur, voire un explorateur, féru de géographie. C'est un lieu fantasmatique où je peux laisser aller l'imagination. L'imaginaire et le réel sont tissés de façon à ce qu'on ne puisse jamais savoir si le lieu est vrai ou s'il est irréel. D'ailleurs, les nouvelles du Pont du Gard vu de nuit, ce sont deux extraits du roman, lequel est un peu bâti sur le modèle de la séquence. J'avais lu La terrasse des audiences au moment de l'adieu d'Yves Navarre et j'avais adoré ce genre de livre qu'on peut ouvrir en plein milieu pour intercepter le début d'un récit qui se déploie en quelques paragraphes, quelques pages, pour conduire à un nouveau récit, également autonome, comme dans le Décaméron. Écrire pour le genre romanesque me paraît beaucoup plus abstrait que faire une pièce. Sans compter que l'acte de réception, chez le lecteur, est différé par rapport à celui du spectateur. Au théâtre, tout est dit dès le lendemain d'une première, tout se passe dans la synchronie où celui qui donne entend l'écho de celui qui reçoit. Ce qu'on dit d'une pièce longtemps après sa création est généralement dans le sillon des choses dites et écrites lors des représentations. Avec le livre, je trouve qu'il est difficile d'agréer des commentaires échelonnés sur des mois après la parution.

V et I: Depuis vos premiers textes, on remarque une présence constante d'un autre texte, d'un texte emprunté. D'une part, le texte-personnage où les références sont clairement identifiées ou identifiables (les poèmes de Nelligan dans Rêve d'une nuit d'bôpital); d'autre part, l'emprunt plus intégré (l'Euvre de Danielle Sarrera, dans la pièce éponyme, devenue plus tard Fêtes d'automne). Le texte d'emprunt précède-t-il votre création ou en procède-t-il?

N. C.: Les deux, c'est selon. L'un ne va pas sans l'autre. Toute construction procède toujours d'une base, et ce qui apparaît être le texte inaugural, un texte déjà publié par exemple, sert de moteur à l'élaboration de la pièce tout entière. Forcément, ce texte de base est lacunaire, afin de permettre une série d'interprétations, d'improvisations, de variantes, lesquelles effectuent une restauration du texte initial, en sorte qu'on ne sait plus, au bout du compte, où est la vérité et où est le mensonge. Le théâtre permet ce jeu plus que tout autre genre: on n'a qu'à penser aux textes de Shakespeare tels qu'ils nous sont retransmis par des générations d'acteurs, qui ont tous ajouté ou modifié des répliques puisqu'ils étaient dépositaires de leurs rôles, et donc transmetteurs du texte qu'on attribue à Shakespeare. Pour ma part, les "Mémoires" de Charles Charles, la carte postale dans Petit Navire, le tableau de Zoé dans La société de Métis, la partition de Don Carlo qu'il faut écrire en 48 heures sous la menace d'un revolver, c'est un peu l'inscription du destin de mes personnages. Je n'écrirai qu'une variation sur ce destin. Mon titre le plus explicite en ce sens est bien Fragments d'une lettre d'adieu lus par des géologues.

V et I : Attardons-nous encore un peu aux autres auteurs. Quand vous écrivez des textes dans lesquels de grands créateurs deviennent vos 
personnages (Nelligan, Verdi), vous vous tenez toujours loin de l'étude biographique. Croyez-vous que le théâtre biographique, comme chez Jovette Marchessault, puisse se transformer trop facilement en piège?

N. C. : C'est une question à laquelle j'ai beaucoup réfléchi. Effectivement, Jovette Marchessault a eu le courage de ne pas maquiller cette voix d'accès à l'écriture. Ce procédé lui appartient. Je voulais pour ma part m'intégrer de façon différente dans les "biographies", en admettant que la vérité sur un autre créateur est toujours, au fond, à l'intérieur de soi. Le fait d'appeler un personnage Violette Leduc, ou de nommer le héros de Rêve d'une nuit d'hôpital Émile Nelligan, est une didascalie comme une autre, c'est-à-dire une indication au lecteur et à l'interprète, une invitation à la référence, et, dans les cas qui nous occupent, à la légende. Autrement, ma première pièce ne serait qu'un adieu à l'adolescence. Après avoir rangé le dossier Nelligan, je me souviens de m'être emporté contre la connerie, ce coup d'encens pour la névrose, cette idolâtrie pour le poète rendu génial par son propre abrutissement. J'ai repris le personnage du poète fou dans Provincetown Playbouse. Mes deux premières pièces, écrites à partir de textes de poètes aujourd'hui disparus, me montraient une voie plus personnelle. Celle de Charles Charles et de ses mémoires inventés par moi. Je suis revenu dix ans plus tard avec un sujet biographique, celui de Verdi, avec Je vous écris du Caire. La pièce, à l'instar de ce qu'elle raconte, était une commande. Je vous écris du Caire n'a pas été un coup très très solide dans mon parcours, mais c'était nécessaire qu'elle soit là. La raison pour laquelle je crois que c'est une pièce qui fonctionne moins bien que les autres tient au fait que mon amour de Verdi a pris le dessus sur l'exploration que je me proposais d'en faire. C'est comme si je n'avais pas réussi à amener Verdi dans ma pièce. Je suis resté en dehors de Verdi. J'ai fait un personnage qui n'a rien à voir avec Verdi, de sorte que c'est une fausse scène, un faux témoignage.

V et I: Dire que la musique occupe une grande place dans votre œuvre est un euphémisme. En plus de mettre en scène la création d'un opéra de Verdi, vos textes sont construits comme une messe, un quatuor, un requiem, un Stabat Mater, etc. Au-delà de l'architecture musicale, jusqu'où la musique (sur les plans formel, thématique, rhétorique) joue-t-elle un rôle dans votre composition littéraire?

N. C. : Le rôle de la musique est prépondérant dans mon écriture. Je pense tout simplement que ce n'est pas voulu. J'ai une formation de musicien et mes repères culturels sont beaucoup plus riches en musique qu'en littérature. Je crois être un musicien qui n'a pas travaillé le matériau approprié pour s'exprimer, et qui a donc découvert à son insu une langue hybride. Souvent les mots ont un sens différents de ce qu'ils veulent dire selon le dictionnaire, parce que leur musique les rapproche d'un mot qui veut dire autre chose. Mon théâtre fonctionne beaucoup là-dessus et ce n'est pas commode pour les metteurs en scène ni pour les acteurs. Les 
Stabat Mater en sont un exemple. Et je dois sans cesse me rappeler que le thêâtre est une chose, que la musique en est une autre.

V et I : La structure musicale est-elle une façon de briser le récit théâtral traditionnel ou du moins un moyen de proposer une nouvelle forme dramatique?

N. C. : Pour moi, le récit théâtral n'est qu'un accessoire de convention. Cela dit, j'ai commis certaines infractions qui m'ont été reprochées en sorte que j'ai dû réfléchir à une question qui n'est pas simple. Question de loi, de permission, et de liberté par rapport au genre théâtral. Je savais que je n'avais pas le droit de dire que Fragments d'une lettre d'adieu lus par des géologues était une pièce policière alors qu'elle s'achève par un monologue sur la question de l'âme, qui ne propose aucune solution à l'intrigue d'abord annoncée. Je ne l'ai peut-être pas dit, mais je l'ai fait. À partir de Fragments, je me suis souvenu de cette phrase de Monique Lepage: "Il y a des lois au théâtre." En effet, si on utilise un matériau aussi archaïque que le théâtre, c'est évident qu'il faut avoir le respect des lois. C'est ce qui m'attire aussi dans la création. On ne fait rien sans le respect des lois.

V et I : Et si on ne les transgresse jamais?

N. C.: $\mathrm{Si}$ on ne les transgresse jamais, on ne prend jamais de risques. Fragments a été mon grand risque. Ce n'était pas de mettre en scène le discours scientifique qui constituait un risque, mais bien de proposer une structure à l'américaine, une pièce à interrogatoire, pour déboucher sur un discours essentiellement poétique. Je faisais le pari que ce pourrait être aussi fort qu'une résolution comme il y en a une dans Provincetown Playhouse. Or, ça a été plus fort. C'est, de toutes mes pièces, celle qui fait le meilleur lien entre la tendance dramatique et la tendance musicale, comme sur un axe qui permettrait au concret de se "spiritualiser". Quand j'écris, je cherche à reproduire ce que me fait la beauté de la musique. C'est uniquement un rapport à la beauté. Il m'arrive de rêver qu'on reçoive un de mes textes comme moi, quand je vais à l'OSM, je reçois une symphonie de Mahler. C'est de l'ambiance. C'est ma façon de dire: écoutez, l'histoire, de toute façon, ne fonctionne pas. En musique, l'histoire ne fonctionne pas. C'est ce qui fait que la musique est belle. C'est là où je me piège aussi, je prête le flanc à beaucoup de noninterprétation. Mes Stabat Mater ne portent pas la mention "théatre " parce que pour moi ce n'est pas du théâtre; raison de plus pour'demander à des comédiennes d'en dire les textes. Ce sont des fragments, des exercices, du reste c'est un projet que j'aime parce qu'il resterait éternellement inachevé, imparfait, voilà pourquoi ils sont chiffrés, ils peuvent s'arrêter comme ils peuvent continuer. C'est le propre des symphonies, des concertos, des séries. J'ai l'impression que les gens sont plus ouverts qu'autrefois face à cette liberté de confondre les genres. Il y a les purs et 
durs qui associent la fin du théâtre avec la chute du rideau. Ils n'ont pas tort. Mais je n'ai pas tort non plus de penser que si pour moi le théâtre commence bien avant un soir de première, il peut s'achever ailleurs, dans l'intimité d'une chambre d'actrice qui se souvient d'un monologue qu'elle a pu dire autrefois.

V et I: La traduction prend de plus en plus de place dans votre travail, notamment l'œuvre de Shakespeare. J'aimerais toutefois m'attarder à un cas plus particulier: Les reines. Beaucoup de choses ont été dites à ce sujet. Serait-il possible de clarifier un peu la situation. Les reines ont-elles été, comme l'écrit Paul Lefebvre dans la préface du livre, "arrachées au Richard III de Shakespeare"?

N. C.: Le matériau de base pour Les reines m'a toujours semblé être la langue. Ce n'est pas la référence à Richard III de Shakespeare qui m'avait donné le souffle nécessaire, mais bien l'hypothèse d'une langue qui soit inscrite dans la sauvagerie de l'ambiance, en 1483. Ce qui est moderne en 1483, c'est aussi moderne qu'aujourd'hui, aussi contemporain, c'est-à-dire qu'on parle de l'acier dans Les reines, on parle de toute la vie qui est en train de changer. Il y a ce côté archaïque du langage qu'il faut travailler avec un ciseau, presque. Pour une raison bien simple, cette langue-là est la seule chose qui vienne suppléer à l'absence de traces. Il n'y a rien, sauf les textes de Shakespeare, pour nous parler de l'Angleterre de 1483. Aucune autre documentation sur la vie quotidienne, sur la vie politique et économique, c'est encore un Moyen Âge de convention, et c'est ce rien-là qui est devenu le centre de ma recherche.

V et I : Les reines (publiée en 1991): une distribution entièrement féminine; même chose pour les textes les plus récents: Stabat Mater I et Stabat Mater II, Les conférencières, texte inédit, et Le petit Köchel, qui sera présenté en Avignon à l'été 2000. Comment expliquez-vous cet acharnement à trouver une parole féminine originale? Parole qui me semble commencer avec le très beau monologue de Carla van Saikin dans Fragments, qui demeure pourtant assez traditionnel du point de vue du discours des femmes.

N. C.: Il y a plusieurs réponses, toutes en noir et blanc. Je ne crois pas vouloir trouver une parole féminine "originale". Mon discours, d'un point de vue émotif, me semble trouver un peu plus de justesse quand c'est une femme qui le dit. L'homme, dans notre société contemporaine, n'est pas le porte-parole de l'émotion. Chaque fois qu'un personnage masculin d'Albee ou de Miller vient pleurer sur scène (c'est vrai aussi pour les personnages masculins de Dubé), on a toujours affaire à un prototype exceptionnel qui vient justement rehausser la légendaire absence d'émotivité de l'homme. Il y a là quelque chose dont l'efficacité m'agace. L'idée de placer devant une masse de géologues une femme au discours contrastant, quoique traditionnel, était ma façon d'interroger cette 
impasse. Je parlerais d'une passation de pouvoir qui a débouché sur Les reines et tout ce qui s'ensuit. Évidemment, il y a aussi l'éternelle question musicale. La voix de femme sans son contraste masculin est intéressante, sur le strict plan de l'audition. Et puis on ne fait jamais les choses pour une seule raison. Il y a une part de coïncidence, c'est peut-être une période. Chose certaine, ces pièces n'ont pas pour but de me cantonner dans un univers exclusif; j'entrevois avec intérêt de nouveaux personnages masculins. J'aimerais aussi écrire une pièce qui mette en scène des vieillards. Pour plus tard. L'idée de vieillir avec mes personnages me plait.

V et I : Le rapport à la scène n'a pas toujours été facile pour vous. Certains metteurs en scène - n'ayons pas peur des mots - ont à l'occasion "détruit "vos textes à la représentation. Avez-vous déjà senti qu'un metteur en scène vous avait trompé, voire trahi, pour reprendre le mot de Kundera? Par ailleurs, avez-vous été attiré par la mise en scène, comme Beckett, qui voulait s'assurer de l'interprétation juste de ses textes?

N. C.: Je ne dirais pas que j'ai trouvé ça difficile. Je n'ai que de beaux souvenirs. Pour moi, ce sont des années d'apprentissage. J'ai toujours intimement associé le plaisir de la vie avec le métier; je ne peux pas avoir de regrets. Se sentir trahi est un sentiment quotidien quand on est dans la création. On se sent trahi par nos proches, nos meilleurs amis, nos plus fidèles exégètes. La critique dans mon cas a tellement mis l'accent sur la difficulté de mettre mes textes en scène que j'ai peut-être lâchement placé mes énergies ailleurs. J'étais d'accord pour le fond. Une chose est certaine: ça ne se passe pas dans un rapport d'amertume ou de tristesse. Ce n'est pas dans mon tempérament d'en vouloir à un metteur en scène, même si, plusieurs fois, j'ai avancé des idées qu'on aurait dû écouter en cours de répétition. Mais il m'est également arrivé de donner de mauvais conseils. J'ai failli faire de la mise en scène et je suis content de ne pas en avoir fait. Qui m'aurait fait confiance? Il faut de l'autorité. Or je n'en ai aucune: je suis trop fin. Puis je ne suis ni Kundera ni Beckett. Je m'inscris dans un paysage américain, québécois et français, sorti d'aucune école de théâtre, d'aucun atelier d'écriture, avec d'énormes références culturelles, des amours mal assorties de classicisme et de postmodernisme, je suis réfractaire à l'actualité et aux médias électroniques, alors c'est tout à fait normal qu'on ne sache pas comment apprivoiser mes choses. À l'époque où je publiais mes textes avant qu'ils soient joués, les gens qui connaissaient déjà la pièce m'en parlaient d'une façon pourtant satisfaisante. Avec Denis Marleau, j'ai trouvé un metteur en scène très complice, en tout cas muni d'une intelligence qui comprend mon écriture, et l'invite à se préciser. Mais ça m'a pris vingt ans...

V et I : Sur scène, vos textes ont parfois enchanté les spectateurs, la critique, et souvent déconcerté les mêmes spectateurs, la même critique. Croyez-vous que cela tienne en partie au caractère non psychologique de vos pièces, à l'opposé du téléroman? 
N. C. : Mes pièces sont très psychologiques. Ces personnages-là ne s'expliquent que par une observation de la psychologie humaine. Il s'agit plutôt du caractère non naturaliste de mon écriture. C'est bien important de faire la nuance. Pour moi, c'est le téléroman qui ne fonctionne pas sur le plan psychologique; Le passage de l'Indiana me paraît beaucoup plus vraisemblable que les histoires organisées psychologiquement pour que le plus grand nombre les comprenne. La langue télévisuelle, qui se veut de bon aloi, me paraît tout aussi, et sinon plus fabriquée que ma langue dite "littéraire". Je dois fournir beaucoup d'effort pour croire aux téléromans, et ça alerte beaucoup mon esprit moqueur!

$\mathbf{V}$ et I : Je tiens à préciser une chose : en parlant du caractère non psychologique, j'ai peut-être donné l'impression d'être méprisant ou de ne pas bien comprendre; je ne veux pas dénigrer la psychologie. C'est plutôt la psychologisation - je crois que le mot est juste - qui causerait un problème. Par exemple, les comédiens ont parfois tendance à trop psychologiser leur personnage.

N. C. : Les metteurs en scènes que je fréquente sont tous des gens qui $a$ priori ont parfaitement compris comment il faudrait monter le texte. Pourtant, on revient inévitablement à des solutions traditionnelles, qui souvent banalisent le propos. Devant le résultat, force est de constater que la psychologisation a pris le dessus. Pourquoi? Je crois que cela fait partie de ma façon d'écrire. Isolez certaines répliques, elles sont d'une banalité phénoménale. Juxtaposez-les, et ça devient injouable. Injouable, voilà bien le mot avec lequel je vis depuis vingt ans! Admettez qu'il faut le faire! Cela dit, j'avoue que les metteurs en scène ont souvent cédé à la tentation de rendre naturalistes des scènes qui ne le sont pas, et qu'inversement, bien peu d'entre eux ont pris mon humour pour exactement ce qu'il devrait être, soit tout simplement de l'humour. On y cherche trop souvent un sens profond, métaphysique.

V et I: Un aspect de plusieurs de vos textes me fascine: le dédoublement du personnage (Charles Charles dans Provincetown Playbouse, Verdi dans Je vous écris du Caire, Joa dans Fêtes d'automne). Qu'est-ce qui vous incite à sonder la question complexe de l'identité, du moi, de cette façon?

N. C.: Dans La société de Métis, Zoé Pé dit: "Deux oreilles, deux bras, deux jambes. Deux mains, deux pieds. L'humanité est faite en double." En réponse à la réplique de l'aveugle: "C'est étrange. On dit "bon pied, bon œil"; mais pourtant, nous avons deux yeux. "Je ne connais personne qui n'ait de fascination pour le dédoublement. Le double est un excellent sujet quand on écrit, quand on crée de façon générale. Le dédoublement, l'effet-miroir est une source intarissable depuis que l'art existe. Je ne crois pas pour autant avoir une fascination personnelle plus grande que la moyenne pour ce mystère, en tout cas pas dans ma vie personnelle. Cela dit, je ne nie pas l'omniprésence de cette fascination dans mes pièces. 
Elle vient toute seule, et j'y fais très attention, surtout depuis Je vous écris $d u$ Caire, où tous les personnages se découvrent un double. Dans Le petit Köchel, j'ai fait deux couples de sœurs, deux interprètes, deux musicologues. En réalité, c'est le chiasme qui m'intéresse plus que le double; c'est-à-dire toutes les possibilités de croix. Jamais je ne suis parti du double pour écrire une pièce. Le double, c'est un repère culturel. Je n'étais pas fier du double dans Je vous écris du Caire, parce qu'il ne fonctionne pas. Le texte a peut-être été une réflexion sur le double, mais la pièce est une affaire inachevée. Mais je ne vais pas passer ma vie à le dire!

V et I : En évoquant tout à l'heure le nom de Kundera, je n'ai pas pu m'empêcher de penser à son idée sur les petites nations, qui étouffent parfois leurs créateurs. Vos récents succès à l'étranger (en France en particulier) vous donnent-ils envie de mettre l'accent davantage sur une carrière internationale?

N. C. : Être étouffé par le Québec, c'est impensable. Le Québec me nourrit énormément dans ses failles. Et la France me paralyse continuellement quand j'y vais dans son désabusement culturel où tout devient politique, tout devient matière à polémique; ça emprisonne beaucoup plus un créateur. C'est impossible de ne pas avoir d'opinion en France; je revendique le droit de ne pas avoir d'opinion si je n'en ai pas. De plus, la mentalité française, surtout chez les intellectuels, encourage non seulement qu'on ait une opinion, mais demande qu'elle soit dite haut et fort et à répétition. Quoi qu'il en soit, je ne pense pas qu'un artiste ait tout contrôle sur la culture de l'endroit d'où il provient, mais je ne crois pas non plus que la culture d'un pays puisse avoir le contrôle sur un artiste. Par ailleurs, ce n'est pas mon propos d'aller défendre le pauvre artiste. Il y a des choses très sombres dans ce que j'écris, comme tout à coup il y a des lumières qui m'échappent; c'est quand même le Québec, dans une large part, qui me renvoie cette lumière. Le milieu artistique m'a soutenu avec beaucoup de générosité.

V et I : Vous dites qu'il y a des choses sombres dans ce que vous écrivez, mais on y trouve aussi des choses plus gaies, même si, en général, on n'associe pas immédiatement vos textes et l'humour. Certains textes contiennent des passages humoristiques ou des répliques très drôles. Vous disiez précédemment que les metteurs en scène n'avaient pas toujours compris votre humour. Quelle place l'humour occupe-t-il exactement dans votre dramaturgie?

N. C. : Pour moi, l'humour est quelque chose de bien important; je suis quelqu'un qui aime rire. Le théâtre est l'art du contraste et de la surprise. L'humour est une façon d'organiser la surprise, l'inattendu. Mais pas nécessairement avec un matériau réputé drôle. Je suis perplexe quand je constate que ce qu'on appelle communément les gags, surtout dans notre 
tradition américaine, sont si efficaces auprès des masses. Le recours à la vulgarité pour susciter des réactions sonores dans un auditoire, qu'on soit au théâtre ou ailleurs, me paraît toujours infantilisant. Dans mes pièces, le moteur de l'humour est souvent la contradiction. J'ai plusieurs fois remarqué qu'on rit de manière inconfortable, presque à la dérobée, quand on écoute mes pièces. Cet inconfort m'amuse. Encore que j'aimerais bien, en fin de vie, écrire une comédie où l'on rirait tout le temps. J'ai un grand culte du rire. Sur le strict plan de l'écriture, je suis toujours à l'affût de la petite chose. J'ai toujours beaucoup de peine quand je vois qu'un metteur en scène a complètement évacué l'humour d'un texte.

V et I : Dans La cobérence fautive, j'ai écrit que "la récurrence des commentaires métatextuels dans Scènes d'enfants transforme le récit en un véritable essai de poétique dramaturgique. "Avez-vous été tenté par l'écriture d'une sorte d'autopoétique, un peu comme Ionesco l'avait fait avec ses Notes et contre-notes ou Kundera avec L'art du roman?

N. C.: Oui mais ça me paraît si laborieux! Je serais attiré par un travail de réflexion non pas sur la façon dont on devrait faire le théâtre, mais bien sur ce que j'aurai accumulé d'observations dans mon métier quant à ce qui cloche au théâtre. Mais au sens où l'ont fait Kundera et Ionesco, non. Je suis mal à l'aise avec l'idée d'écrire sur mon œuvre.

V et I : Robert Lévesque a écrit dans l'un de ses carnets ${ }^{1}$ que vous étiez, sur le plan politique, insaisissable. Est-ce bien vrai? Si oui, est-ce délibéré?

N. C.: Tout ce qui peut sortir de ma plume et qui peut avoir un impact sur quelque chose qui me répugne de dire, je ne l'écrirai pas. C'est vrai que politiquement je suis insaisissable parce que le pouvoir n'est pas en politique. Lorsque j'essaie moi-même de m'interroger sur mes opinions politiques, je me dérobe et je pense à autre chose. Le pouvoir est dans l'individu, et j'ai le droit de me désintéresser totalement d'une actualité qui depuis vingt ans me déprime. On m’a déjà aussi dit qu'il y avait un comportement de fuite dans mon écriture. Fuir est à peu près la seule chose qui m'intéresse dans la vie. Si c'était un reproche, je suis bien prêt à revoir cet aspect par trop imprécis de mon discours. Mais si c'était un constat, rien de plus juste. Fuir est obéir à un mouvement. C'est toujours en déplacement. Quand on dit une chose, on pense aussi au contraire de la chose; le plaisir est de confronter la chose, son contraire, et de trouver tout à coup comment un troisième élément va trouver une place dans ce que l'on a à dire. Dans mon cas, le contenu a sans doute son importance, mais la forme est ce qui m'intéresse davantage. Pour moi, l'art n'a pas de raison d'être, si ce n'est que du message ou du discours. Pour avoir

1. La liberté de blâmer. Carnets et dialogues sur le théâtre, Montréal, Boréal, coll. "Papiers collés", 1997, p. 38. 
abordé la politique comme sujet de dialogues dans quelques-unes de mes pièces (dans Petit navire, un peu dans Je vous écris du Caire, et aussi dans Stabat Mater II), je sais à présent que ce sujet ne m'inspire rien de tangible. Je voudrais bien que la politique retrouve ses lettres de noblesse, mais c'est impossible dans un contexte aussi médiatisé que le nôtre. Il existe pourtant de très belles pièces politiques, je pense à $L a$ jeune fille et la mort, qui pour moi est un sommet. Qu'est-ce qu'on a tant vécu ici pour écrire des pièces politiques qui pourraient se comparer au répertoire des pays de l'Est ou du théâtre sud-américain? On n'est même pas allé au Vietnam, sinon qu'en regardant des films américains.

Peut-être qu'en Tchécoslovaquie, en 1968, j'aurais aimé être parmi les intellectuels qui ont participé au Printemps de Prague. Peut-être qu'en France j'aurais vécu une chose exaltante durant mai 68. Mais j'ai choisi de venir au monde au Québec avant et après l'an 2000, où le radotage est tel qu'il ne permet même pas une désillusion salutaire pour mon imagination. D'autres y ont puisé de l'inspiration. Au fond, j'écris sur des choses que j'ai envie d'explorer ou d'approfondir. Pas sur une matière qui m'est familière. La seule chose qui m'intéresse, en réalité, c'est de commencer un texte pour voir ce qu'il va dire, et comment. 